Short Communication

\title{
On Minimally Nonouterplanarity of the Semi Total (Point) Graph of a Graph
}

\author{
V. R. Kulli ${ }^{1}$ and K. M. Niranjan ${ }^{2}$ \\ ${ }^{1}$ Department of Mathematics, Gulbarga-585106, Karnataka, India \\ ${ }^{2}$ Department of Computer Science and Engineering, S.J.M.I.T, NH4 Bye Pass Road, \\ Chitradurga-577502, Karnataka, India
}

Received 17 March 2009, accepted in final revised form 4 July 2009

\begin{abstract}
We present here characterizations of graphs whose semi total (point) graphs are outerplanar and $k$-minimally nonouterplanar $(k=1,2$ or 3$)$.

Keywords: Semi total (Point); Minimally nonouterplanarity; Outerplnanar; Block.

CC 2009 JSR Publications. ISSN: 2070-0237 (Print); 2070-0245 (Online). All rights reserved.

DOI: $10.3329 /$ jsr.v1i3.2578

J. Sci. Res. 1 (3), 551-557 (2009)
\end{abstract}

\section{Introduction}

All graphs considered are finite, undirected and without loops or multiple lines. For standard terminology and notations we follow Harary [1]. The semi total (point) graph $T_{2}(G)$ of a graph $G$ is the graph whose point set is $V(G) \cup X(G)$ where two points are adjacent if and only if (i) they are adjacent points of $G$, or (ii) one is a point and the other is a line of $G$ incident with it. This concept was introduced by Sampathkumar and Chikkodimath [2, 3]. In 1975 Kulli [4] introduced the concept minimally nonouterplanar graph. The inner point number $i(G)$ of a planar graph $G$ is the minimum possible number of points not belonging to the boundary of the exterior region in any embedding of $G$ in the plane. Obviously $G$ is outerplanar if and only if $i(G)=0$. A graph $G$ is minimally nonouterplanar if $i(G)=1$, and $G$ is $k$-minimally $(k \geq 2)$ nonouterplanar if $i(G)=k$.

Definition 1.1: A block of a graph $G$ is a maximal nonseperable subgraph.

Definition 1.2: A line joining two nonadjacent points of a cycle is called a chord of the cycle.

\footnotetext{
${ }^{2}$ Corresponding author: niru_km@yahoo.com
} 
Definition 1.3: Let $C$ be a cycle with $p \geq 4$ points. If a path of length two joins two nonadjacent points of $C$ then it is called a theta ${ }^{+}$block.

The following will be useful in the proof of our results:

Theorem A [2]: For a graph $G$, let $T_{2}(G)$ be the semi total (point) graph of $G$. Then $T_{2}(G)$ is planar if and only if $G$ is planar.

\section{Prerequisites}

We first prove two lemmas which are useful to prove our results.

Lemma 1: If $G$ is a cycle with $n(n \geq 1)$ chords, such that no two chords intersect when all points lie on the exterior region, then $i\left(T_{2}(G)\right)=n$.

Proof: To prove the result we use mathematical induction on $n$.

Suppose $n=1$. Then graph $G$ is a cycle with one chord, when all points lie on the exterior region. Then by Theorem A, $T_{2}(G)$ is planar. On drawing of $G$ in the plane as shown in Fig. 1a, in any plane embedding of $T_{2}(G)$ has one inner point (see Fig. 1b). Hence the result is true for $n=1$.

Suppose $n=2$. Then graph $G$ is a cycle with two chords, such that no two chords intersect when all points lie on the exterior region. Then by Theorem A, $T_{2}(G)$ is planar. On drawing of $G$ in the plane as shown in Fig. 2(a), in any plane embedding of $T_{2}(G)$ has two inner points (see Fig.2b). Hence the result is true for $n=2$.

Assume the result is true for $n=m$ chords.

Now we prove the result for $n=m+1$ chords. Let $e_{j}$ be the chord of $G$. Delete from $G$ the chord $e_{j}$, let $G^{\prime}=G-e_{j}$, which has $\mathrm{m}$ chords. Then by inductive hypothesis, $i\left(T_{2}\left(G^{\prime}\right)\right)=m$.

Now again rejoin the chord $\mathrm{e}_{\mathrm{j}}$ to $G^{\prime}$, resulting the graph $G$, which produces an inner point in $i\left(T_{2}(G)\right)$. On drawing of $G$ in the plane as shown in Fig. 3a, in any plane embedding of $T_{2}(G)$ has $m+1$ inner points. (see Fig. 3b). Hence the result.

Lemma 2. If $G$ is a theta ${ }^{+}$block, then $i\left(T_{2}(G)\right)=3$.

Proof. Suppose $G$ is a theta ${ }^{+}$block. Then by the definition of theta ${ }^{+}$block, $G$ contains path of length two joining a pair of nonadjacent points. Then by Theorem A, $T_{2}(G)$ is planar. On drawing of $G$ in the plane as shown in Fig.4(a), in any plane embedding of $T_{2}(G)$ it has three inner points (see Fig.4b). Thus $i\left(T_{2}(G)\right)=3$.

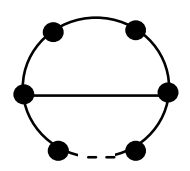

(a)
Fig.1.

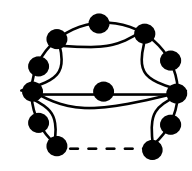

(b) 


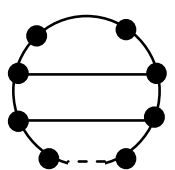

(a)

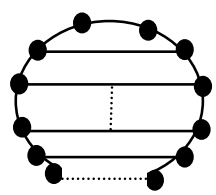

(a)

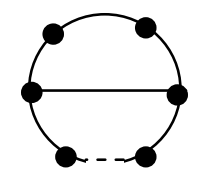

(a)

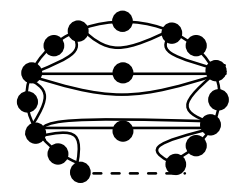

(b)
Fig. 2.

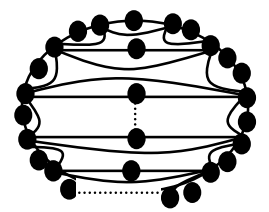

(b)

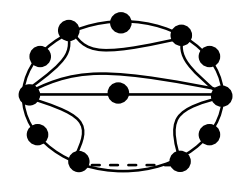

(b)

\section{Main Results}

Theorem 2 in the paper [4] is incorrect. Theorem 2 of [4] states that for a graph $G$, let $T_{2}(G)$ be the semi total (point) graph of $G$. Then $T_{2}(G)$ is planar (outerplanar) if and only if $G$ is planar (outerplanar). As per the above theorem statement graph shown below (see Fig 5), now $G$ is outerplanar but $T_{2}(G)$ is not a outerplanar.Therefore Theorem 2 in the paper [4] is incorrect.

G:

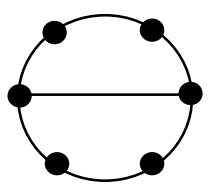

$T_{2}(G):$

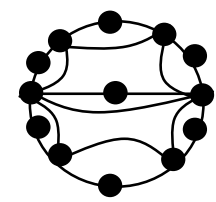

Fig. 5.

Now we improve the above stated theorem for outerplanarity.

Theorem 1: The semi total (point) graph $T_{2}(G)$ of a graph $G$ is outerplanar if and only if every block of $G$ is either a line or a cycle. 
Proof: Suppose $T_{2}(G)$ is outerplanar. Then it is planar and by Theorem A, $G$ is planar, which implies that every block of $G$ is planar. Assume $G$ has a cyclic block $b_{i}$ other than cycle. Then the block $b_{i}$ has a subgraph homeomorphic from $K_{4}-\chi$. Let $G^{\prime}=K_{4}-\chi$, which has a chord, by Lemma $1, i\left(T_{2}\left(G^{\prime}\right)\right)=1$. Therefore $T_{2}(G)$ has at least one inner point. Thus $i\left(T_{2}(G)\right) \geq 1$. Hence $T_{2}(G)$ is nonouterplanar, a contradiction. Thus every block of $G$ is either a line or a cycle.

Conversely, suppose every block of $G$ is either a line or a cycle. Then it is easy to see that, $T_{2}(G)$ is outer planar. This completes the proof of the theorem.

We now characterize graphs whose semi total (point) graphs are minimally nonouterplanar.

Theorem 2: The semi total (point) graph $T_{2}(G)$ of a graph $G$ is minimally nonouterplanar if and only if $G$ has exactly one block such that it is a cycle with one chord, when all point lie on the exterior region and every other block of $G$ is either a line or a cycle.

Proof: Suppose $T_{2}(G)$ is minimally nonouterplanar.Then by Theorem A, $G$ is planar. We now consider the following cases.

Case 1. Suppose every block of $G$ is either a line or a cycle. Then $G$ is an outerplanar graph and by Theorem $1, T_{2}(G)$ is outerplanar, a contradiction.

Case 2. Suppose $G$ has exactly one block which is a cycle with at least two chords, such that no two chords intersect when all points lie on the exterior region and every other block of $G$ is either a line or a cycle. Then by Lemma $1, i\left(T_{2}(G)\right) \geq 2$, a contradiction.

Case 3. Suppose $G$ has exactly one block such that it is a theta ${ }^{+}$block and every other block of $G$ is either a line or a cycle. Then by Lemma 2, $i\left(T_{2}(G)\right)=3$, a contradiction.

Case 4. Suppose $G$ has exactly two blocks each of which is a cycle with at least one chord, when all points lie on the exterior region and every other block of $G$ is either a line or a cycle. Then by Lemma $1, i\left(T_{2}(G)\right) \geq 2$, a contradiction.

We have exhausted all possibilities. In each case we found that $T_{2}(G)$ is not minimally nonouterplanar. Thus we conclude that $\mathrm{G}$ holds the condition.

Conversely, suppose $\mathrm{G}$ has exactly one block such that it is a cycle with one chord, when all points lie on the exterior region and every other block of $G$ is either a line or a cycle. Then by Lemma $1, i\left(T_{2}(G)\right)=1$, since every other block of $T_{2}(G)$ is outerplanar.Hence $T_{2}(G)$ is minimally nonouterplanar.

In the following theorem, we establish a characterization of graphs whose semi total (point) graphs are 2-minimally nonouterplanar.

Theorem 3: The semi total (point) graph $T_{2}(G)$ of a graph $G$ is 2-minimally nonouterplanar if and only if $G$ holds (1) or (2):

(i) $G$ has exactly one block which is a cycle with two chords, such that no two chords intersect when all points lie on the exterior region and every other block of $G$ is either a line or a cycle.

(ii) $G$ has exactly two blocks each of which is a cycle with one chord, when all points lie on the exterior region and every other block of $G$ is either a line or a cycle. 
Proof: Suppose $T_{2}(G)$ is 2-minimally nonouterplanar.Then by Theorem A, $G$ is planar. We now consider the following cases.

Case 1. Suppose every block of $G$ is either a line or a cycle. Then $G$ is an outerplanar graph and by Theorem $1, T_{2}(G)$ is outerplanar, a contradiction.

Case 2. Suppose $G$ has exactly one block which is a cycle with one chord, when all points lie on the exterior region and every other block of $G$ is either a line or a cycle. Then by Theorem 2, $i\left(T_{2}(G)\right)=1$, a contradiction.

Case 3. Suppose $G$ has exactly one block which is a cycle with at least three chords, such that no two chords intersect when all points lie on the exterior region and every other block of $G$ is either a line or a cycle. Then by Lemma $1, i\left(T_{2}(G)\right) \geq 3$, a contradiction.

Case 4. Suppose $G$ has exactly one block such that it is a theta ${ }^{+}$block and every other block of $G$ is either a line or a cycle. Then by Lemma 2, $i\left(T_{2}(G)\right)=3$, a contradiction.

Case 5. Suppose $G$ has exactly two blocks one of which is a cycle with at least two chords and other is a cycle with at least one chord, such that no two chords intersect when all points lie on the exterior region and every other block of $G$ is either a line or a cycle. Then by Lemma $1, i\left(T_{2}(G)\right) \geq 3$, a contradiction.

Case 6. Suppose $G$ has exactly three blocks each of which is a cycle with at least one chord, when all points lie on the exterior region and every other block of $G$ is either a line or a cycle. Then by Lemma $1, i\left(T_{2}(G)\right) \geq 3$, a contradiction.

We have exhausted all possibilities. In each case we found that $T_{2}(G)$ is not 2-minimally nonouterplanar. Thus we conclude that $G$ holds the conditions.

Conversely, suppose $G$ has exactly one block which is a cycle with two chords, such that no two chords intersect when all points lie on the exterior region and every other block of $G$ is either a line or a cycle. Then by Lemma $1, i\left(T_{2}(G)\right)=2$, since every other block of $T_{2}(G)$ is outerplanar.

Again suppose $G$ has exactly two blocks each of which is a cycle with one chord, when all points lie on the exterior region and every other block of $G$ is either a line or a cycle. Then by Lemma $1, i\left(T_{2}(G)\right)=2$, since every other block of $T_{2}(G)$ is outerplanar.Hence $T_{2}(G)$ is 2-minimally nonouterplanar.

Now we establish a characterization of graphs whose semi total (point) graphs are 3-minimally nonouterplanar.

Theorem 4: The semi total (point) graph $T_{2}(G)$ of a graph $G$ is 3minimally nonouterplanar if and only if

(i) $G$ has exactly one block which is a cycle with three chords, such that no two chords intersect when all points lie on the exterior region and every other block of $G$ is either a line or a cycle, or 
(ii) $G$ has exactly one theta ${ }^{+}$block and every other block of $G$ is either a line or a cycle, or

(iii) $G$ has exactly two blocks one of which is a cycle with two chords and other is a cycle with one chord, such that no two chords intersect when all points lie on the exterior region and every other block of $G$ is either a line or a cycle, or

(iv) $G$ has exactly three blocks each of which is a cycle with one chord, when all points lie on the exterior region and every other block of $G$ is either a line or a cycle.

Proof: Suppose $T_{2}(G)$ is 3-minimally nonouterplanar.Then by Theorem A, $G$ is planar. We now consider the following cases.

Case 1. Suppose every block of $G$ is either a line or a cycle. Then $G$ is an outerplanar graph and by Theorem $1, T_{2}(G)$ is outerplanar, a contradiction.

Case 2. Suppose $G$ has exactly one block which is a cycle with one chord, when all points lie on the exterior region and every other block of $G$ is either a line or a cycle. Then by Theorem 2, $i\left(T_{2}(G)\right)=1$, a contradiction.

Case 3. Suppose $G$ has exactly one block which is a cycle with two chords, such that no two chords intersect when all points lie on the exterior region and every other block of $G$ is either a line or a cycle. Then by Theorem $3, i\left(T_{2}(G)\right)=2$, a contradiction.

Case 4. Suppose $G$ has exactly one block which is a cycle with at least four chords, such that no two chords intersect when all points lie on the exterior region and every other block of $G$ is either a line or a cycle. Then by Lemma $1, i\left(T_{2}(G)\right) \geq 4$, a contradiction.

Case 5. Suppose $G$ has exactly two blocks each of which is a cycle with one chord, when all points lie on the exterior region and every other block of $G$ is either a line or a cycle. Then by Theorem 3, $i\left(T_{2}(G)\right)=2$, a contradiction.

Case 6. Suppose $G$ has exactly two blocks each of which is a cycle with at least two chords, such that no two chords intersect when all points lie on the exterior region and every other block of $G$ is either a line or a cycle. Then by Lemma $1, i\left(T_{2}(G)\right) \geq 4$, a contradiction.

Case 7. Suppose $G$ has exactly two blocks each of which is a theta ${ }^{+}$block and every other block of $G$ is either a line or a cycle. Then by Lemma $2, i\left(T_{2}(G)\right) \geq 4$, a contradiction.

Case 8. Suppose $G$ has exactly two blocks one of which is a cycle with at least three chords and other is a cycle with at least one chord, such that no two chords intersect when all points lie on the exterior region and every other block of $G$ is either a line or a cycle. Then by Lemma $1, i\left(T_{2}(G)\right) \geq 4$, a contradiction.

Case 9. Suppose $G$ has exactly two blocks one of which is a cycle with at least one chord and other is a theta ${ }^{+}$block, such that no two chords intersect when all points lie on the exterior region. Then by Lemma 2 and Lemma $1, i\left(T_{2}(G)\right) \geq 4$, a contradiction.

Case 10. Suppose $G$ has exactly three blocks in which two blocks each of which is a cycle one with at least chord and other is a cycle with at least two chords, such that no two 
chords intersect when all points lie on the exterior region. Then by Lemma $1, i\left(T_{2}(G)\right) \geq 4$, a contradiction.

Case 11. Suppose $G$ has exactly four blocks each of which is a cycle with at least one chord, when all points lie on the exterior region. Then by Lemma $1, i\left(T_{2}(G)\right) \geq 4$, a contradiction.

We have exhausted all possibilities. In each case we found that $T_{2}(G)$ is not 3-minimally nonouterplanar. Thus we conclude that $G$ holds the conditions.

Conversely, suppose $G$ has exactly one block which is cycle with three chords, such that no two chords intersect when all points lie on the exterior region and every other block of $G$ is either a line or a cycle. Then by Lemma $1, i\left(T_{2}(G)\right)=3$, since every other block of $T_{2}(G)$ is outerplanar.

Suppose $G$ has exactly one block which is a theta ${ }^{+}$block and every other block of $G$ is either a line or a cycle. Then by Lemma $2, i\left(T_{2}(G)\right)=3$, since every other block of $T_{2}(G)$ is outerplanar.

Suppose $G$ has exactly two blocks one of which is a cycle with two chords and other is a cycle with one chord, such that no two chords intersect when all points lie on the exterior region and every other block of $G$ is either a line or a cycle. Then by Lemma 1 , $i\left(T_{2}(G)\right)=3$, since every other block of $T_{2}(G)$ is outerplanar.

Suppose $G$ has exactly three blocks each of which is a cycle with one chord, when all points lie on the exterior region and every other block of $G$ is either a line or a cycle. Then by Lemma $1, i\left(T_{2}(G)\right)=3$, since every other block of $T_{2}(G)$ is outerplanar. Hence $T_{2}(G)$ is 3-minimally nonouterplanar.

\section{Conclusions}

We present here characterizations of graphs whose semi total (point) graphs are outerplanar and $k$-minimally nonouterplanar $(k=1,2$ or 3$)$.

We further find characterizations of graphs whose semi total (point) graphs are outerplanar in terms of forbidden subgraphs and k-minimally nonouterplanar $(k=1,2$ or 3$)$ in terms of forbidden subgraphs.

\section{Acknowledgements}

The authors are grateful to the reviewers for their critical comments and valuable suggestions.

\section{References}

1. F. Harary, Graph Theory (Addison Wesley, Reading, Mass, 1969).

2. E. Sampath Kumar and S. B. Chikkodimath, J. Karnatak University Science, 18, 281, (1973).

3. E. Sampath Kumar and S. B.Chikkodimath, J. Karnatak University Science, 18, 285, (1973).

4. V. R.Kulli, Proc. Indian. Nat. Sci. Acad, 41, 275, (1975). 